\title{
Numerical Investigation of Fluid Flow and Performance Prediction in a Fluid Coupling Using Large Eddy Simulation
}

\author{
Wei Cai, ${ }^{1}$ Yuan Li, ${ }^{1}$ Xingzhong Li, ${ }^{2}$ and Chunbao Liu ${ }^{1}$ \\ ${ }^{1}$ School of Mechanical Science and Engineering, Jilin University, Changchun 130022, China \\ ${ }^{2}$ State Key Laboratory of Automotive Simulation and Control, Jilin University, Changchun, China \\ Correspondence should be addressed to Chunbao Liu; liuanbc@126.com
}

Received 27 December 2016; Revised 22 May 2017; Accepted 9 July 2017; Published 21 August 2017

Academic Editor: Funazaki Ken-ichi

Copyright (c) 2017 Wei Cai et al. This is an open access article distributed under the Creative Commons Attribution License, which permits unrestricted use, distribution, and reproduction in any medium, provided the original work is properly cited.

\begin{abstract}
Large eddy simulation (LES) with various subgrid-scale (SGS) models was introduced to numerically calculate the transient flow of the hydraulic coupling. By using LES, the study aimed to advance description ability of internal flow and performance prediction. The CFD results were verified by experimental data. For the purpose of the description of the flow field, six subgrid-scale models for LES were employed to depict the flow field; the distribution structure of flow field was legible. Moreover, the flow mechanism was analyzed using 3D vortex structures, and those showed that DSL and KET captured abundant vortex structures and provided a relatively moderate eddy viscosity in the chamber. The predicted values of the braking torque for hydraulic coupling were compared with experimental data. The comparison results were compared with several simulation models, such as SAS and RKE, and SSTKW models. Those comparison results showed that the SGS models, especially DSL and KET, were applicable to obtain the more accurate predicted results than SAS and RKE, and SSTKW models. Clearly, the predicted results of LES with DSL and KET were far more accurate than the previous studies. The performance prediction was significantly improved.
\end{abstract}

\section{Introduction}

A fluid coupling or hydraulic coupling is a hydrodynamic device commonly used in applications requiring smooth torque transmission. It is called coupling for short, which consists of an input rotor (pump impeller) and a matching output rotor (turbine runner), facing each other with a small gap without mechanical contact. It enables transmitting rotating mechanical power, where it requires variable speed operation and controlled start-up without shock loading of the power transmission system. Fluid couplings can also act as hydrodynamic brakes, named hydraulic retarder, dissipating rotational energy as heat through frictional forces.

The transient flow field in fluid coupling is very representative. The rotor and stator impellers comprised the enclosed fluid chamber, also involved in rotor-stator interaction. The chamber could be partially filled during operation. A number of literatures had demonstrated how complex and unsteady the flow was in fluid coupling (see, for example, McKinnon et al. [1] and Hampel et al. [2, 3]). Actually, there were unsteady and turbulent flows with intense secondary flows in the chamber, which were very difficult to calculate because of the lack of understanding of unsteady turbulent flows. In this study, a numerical investigation was presented using large eddy simulation (LES) to study the flow circulation during energy transmission and predict the external performances. Bai et al. utilized standard $k-\varepsilon$ turbulence model as closure of Reynolds averaged Navier-Stokes (RANS) equations to simulated fluid flow in fluid coupling [4]. Huitenga and Mitra developed a code in the body-fitted rotating coordinates to solve RANS equations with $k-\varepsilon$ turbulence model $[5,6]$. Luo et al. employed SIMPLEC algorithm and SST $k-\omega$ turbulence model to simulate the steady state flows [7]. They also used PISO algorithm and RNG $k$ - $\omega$ turbulence model to calculate the gas-liquid two-phase flows in fluid coupling [8]. Hur et al. applied the realizable $k-\varepsilon$ turbulence model together with the VOF method to investigate two-phase flow of water fluid coupling [9]. Obviously, the RANS method was dominant. However, it was well known that this method had theoretical defects, such as the overprediction of viscosity and the failure 
of describing the dynamic pressure fluctuation. Those defects definitely resulted in prediction errors both in fluid flow and in external performances.

Compared with RANS, LES had essentially theoretical advantage, which directly resolved large-scale eddies. It also could alleviate the serious area of modeling uncertainty and was much less model dependent than RANS. LES had been successfully applied in many industrial flows, either in compressible flow, such as compressors [10] and gas turbine [11], or in incompressible flow, such as Francis turbine [12]. Basically, those publications proved that LES was more accurate in flow calculation. Hence, the motivation of present study came from the current situation of turbulence models applied in computation of fluid coupling which were relatively backward. In this paper, a simplified hydraulic coupling, removing the inlet and outlet, was chosen to carry out the investigation. By using LES, the study aimed to advance description ability of internal flow, especially unsteady flow phenomena. LES simulation results were verified by experimental data. Moreover, the performance prediction was improved. It should be noted that accurate prediction has significant value. And the accuracy was good enough, which would reach a level permitting design solely from computational results instead of experiments.

\section{Numerical Simulation}

2.1. SGS Models. The filtering governing equations for fluid coupling simulations can be written as in the following form:

$$
\begin{gathered}
\frac{\partial \overline{u_{i}}}{\partial x_{i}}=0 \\
\frac{\partial \overline{u_{i}}}{\partial t}+\frac{\partial\left(\overline{u_{i}} \overline{u_{j}}\right)}{\partial x_{j}}=-\frac{1}{\rho} \frac{\partial \bar{p}}{\partial x_{i}}+\mu \frac{\partial^{2} \overline{u_{i}}}{\partial x_{j}^{2}}-\frac{\partial \tau_{i j}}{\partial x_{j}} .
\end{gathered}
$$

The equations are unclosed because of the introduction of $\tau_{i j}$ and require closure by structuring the model. The SGS stress tensor in LES is formulated using the concept of eddy viscosity.

$$
\begin{aligned}
\tau_{i j}-\frac{1}{3} \delta_{i j} \tau_{k k} & =-2 \mu_{t} \overline{S_{i j}}, \\
\overline{S_{i j}} & =\frac{1}{2}\left(\frac{\partial \overline{u_{i}}}{\partial x_{j}}+\frac{\partial \overline{u_{j}}}{\partial x_{i}}\right) .
\end{aligned}
$$

The eddy viscosity $\mu_{t}$, which can be obtained from the SGS model in (3), represents the SGS eddy viscosity. Thus, (3) and (4) and the specific SGS model lead to the closure of (2).

In LES, eddies in the turbulence are divided into largescale and small-scale eddies by the filtering way, which characterize the vortex of energy and dissipation, respectively. Afterwards, large eddies are resolved directly by the instantaneous Navier-Stokes equations, while the small eddies are modeled by virtue of subgrid-scale (SGS) models. SGS models including Smagorinsky-Lilly (SL) model, Dynamic Smagorinsky-Lilly (DSL) model, Wall-Adapting Local Eddy Viscosity (WALE) model, Algebraic Wall-Modeled LES
(WMLES) model, Algebraic WMLES S-Omega (WMLES $S-\Omega$ ) model, and Dynamic Kinetic Energy Transport (KET) model were involved in this study, which were introduced in Table 1.

2.2. Computational Setup and Solution. In this paper, threedimensional models of the hydraulic coupling are shown in Figure 1(a).

The complex geometry of hydraulic elements was accurately represented and the computational meshes were appropriately distributed with ANSYS ICEM to generate the structured hexahedral grid of the entire hydraulic coupling. The intensive type grid was used to refine the boundary layer. Mesh-point clustering was performed near the boundary layer and cell deformity was checked with ANSYS ICEM. Figure 1(b) shows the generation process of the structured grid of the entire passage. The detailed properties of CFD model were summarized in Table 2. Furthermore, the dependence on grid was tested by High Performance Computer (HPC) $(2.60 \mathrm{GHz}, 8 \times 2 \mathrm{CPU})$ under different grid numbers based on the pump rotational speed of $600 \mathrm{rpm}$. The calculation results are shown in Figure 1(c).

\section{Internal Fluid Flow}

3.1. Reynolds Number Configuration. Reynolds number (Re) was introduced to evaluate the flow condition. It was defined as the ratio of inertia force to viscous force:

$$
\mathrm{Re}=\frac{\rho v L}{\mu},
$$

where $L$ was the characteristic length and could be calculated as

$$
L=\frac{4 A}{p},
$$

where $A$ was the sectional area for the flow passage; $p$ was the wetted perimeter length.

Three annular sections perpendicular to the interface along the radial direction were equally extracted out for appropriately studying the flow conditions. Figure 2 describes the distributions of $\mathrm{Re}$ for three axial sections in different turbulence models and R85, R120, and R155, respectively, represented different positions. The position was a ring surface in the annular sections and its radius was $R$. The value of $R$ was $85 \mathrm{~mm}, 120 \mathrm{~mm}$, and $155 \mathrm{~mm}$, respectively.

Re ranged from 0 and 60000, suggesting that the flow within the turbine was mainly in a sufficient turbulent flow conditions. WALE and WMLES models allowed the locally larger Re values at R120 and R155 and the corresponding gradient change was distorted according to the working principle of hydraulic coupling. The flow at the outer ring was more intense and corresponding gradient changes were more obvious. However, WALE and WMLES provided a relatively large $\mathrm{Re}$ values in the near-wall region and laminar flows were transited into turbulence earlier. Although WMLES $S-\Omega$, compared with WALE and WMLES, had the smaller Re values in the near-wall region, the Re value in the mainstream 
TABLE 1: The equations of the six SGS models.

\begin{tabular}{|c|c|c|}
\hline SGS models & Equations & Note \\
\hline SL $[13,14]$ & $\begin{array}{c}|\bar{S}|=\sqrt{2 \bar{S}_{i j} \bar{S}_{i j}} \\
L_{s}=\min \left(\kappa d, C_{s} \Delta\right) \\
\Delta=V^{1 / 3} \\
\mu_{t}=\rho L_{s}^{2}|\bar{S}|\end{array}$ & $\begin{array}{l}L_{s} \text { is the mixing length for subgrid scales; } \\
\kappa \text { is the von Kármán constant; } d \text { is the } \\
\text { distance to the closest wall; } C_{\mathrm{s}} \text { is the } \\
\text { Smagorinsky constant, } 0.1 ; \Delta \text { is the local } \\
\text { grid scale. }\end{array}$ \\
\hline DSL $[15,16]$ & 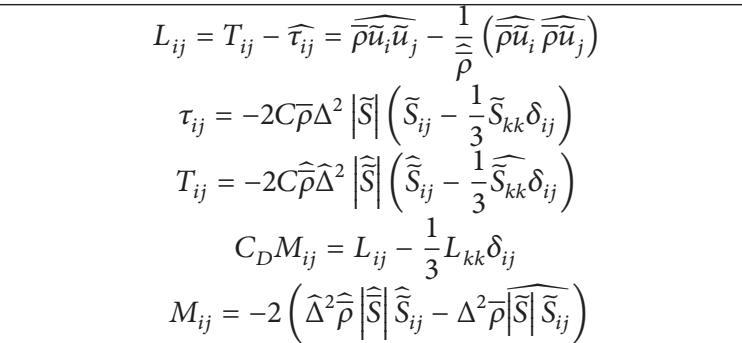 & $\begin{array}{l}\text { The new filter width } \widehat{\Delta} \text { is equal to two } \\
\text { times the grid filter width } \Delta ; T_{i j} \text { is the } \\
\text { SGS stress tensor with } \widehat{\Delta} ; L_{i j} \text { is the added } \\
\text { value of SGS stress tensor with two times } \\
\text { consecutive filtering compared to the } \\
\text { filtering only with } \widehat{\Delta} \text {. }\end{array}$ \\
\hline WALE [14, 16-18] & $\begin{array}{c}\mu_{t}=\rho L_{s}^{2} \frac{\left(S_{i j}^{d} S_{i j}^{d}\right)^{3 / 2}}{\left(\bar{S}_{i j} \bar{S}_{i j}\right)^{5 / 2}+\left(S_{i j}^{d} S_{i j}^{d}\right)^{5 / 4}} \\
L_{s}=\min \left(\kappa d, C_{w} V^{1 / 3}\right) \\
S_{i j}^{d}=\frac{1}{2}\left(\bar{g}_{i j}^{2}+\bar{g}_{j i}^{2}\right)-\frac{1}{3} \delta_{i j} \bar{g}_{k k}^{2} \\
\bar{g}_{i j}=\frac{\partial \bar{u}_{i}}{\partial x_{j}}\end{array}$ & $C_{w}=0.325$ \\
\hline WMLES $[19,20]$ & $\begin{array}{c}v_{t}=\min \left[\left(\kappa d_{w}\right)^{2},\left(C_{\mathrm{smag}} \Delta\right)^{2}\right] \cdot S \cdot\left\{1-\exp \left[-\left(\frac{y^{+}}{25}\right)^{3}\right]\right\} \\
\Delta=\min \left(\max \left(C_{w} \cdot d_{w} ; C_{w} \cdot h_{\max }, h_{\mathrm{wn}}\right) ; h_{\max }\right)\end{array}$ & $\begin{array}{l}d_{w} \text { is the wall distance; } S \text { is the strain rate; } \\
\kappa=0.41, C_{w}=0.15 \text {, and } C_{\text {smag }}=0.2 \text { are } \\
\text { constants; } y^{+} \text {is the normal to the wall } \\
\text { inner scaling. } h_{\max } \text { is the maximum edge } \\
\text { length for a rectilinear hexahedral cell; } \\
h_{\mathrm{wn}} \text { is the wall-normal grid spacing. }\end{array}$ \\
\hline WMLES $S-\Omega$ & $\mu_{t}=(C \Delta)^{2}|S-\Omega|\left\{1-\exp \left[-\left(\frac{y^{+}}{25}\right)^{3}\right]\right\}$ & \\
\hline $\operatorname{KET}[14,21]$ & $\begin{array}{c}k_{\mathrm{sgs}}=\frac{1}{2}\left(\bar{u}_{k}^{2}-\bar{u}_{k}^{2}\right) \\
\mu_{t}=C_{k} \rho k_{\mathrm{sgs}}^{1 / 2} \Delta_{f} \\
\rho \frac{\partial \bar{k}_{\mathrm{sgs}}}{\partial t}+\rho \frac{\partial \bar{u}_{j} \bar{k}_{\mathrm{sgs}}}{\partial x_{j}}=-\tau_{i j} \frac{\partial \bar{u}_{i}}{\partial x_{j}}-C_{\varepsilon} \rho \frac{k_{\mathrm{sgs}}^{3 / 2}}{\Delta_{f}}+\frac{\partial}{\partial x_{j}}\left(\frac{u_{t}}{\sigma_{k}} \frac{\partial k_{\mathrm{sgs}}}{\partial x_{j}}\right) \\
\tau_{i j}-\frac{2}{3} \rho k_{\mathrm{sgs}} \delta_{i j}=-2 C_{k} \rho k_{\mathrm{sgs}}^{1 / 2} \Delta_{f} \bar{S}_{i j}\end{array}$ & $\begin{array}{c}C_{k} \text { is model coefficient; } K_{\text {sgs }} \text { is the } \\
\text { subgrid-scale kinetic energy. } \mu_{t} \text { is the } \\
\text { subgrid-scale eddy viscosity; } \Delta_{f} \text { is the } \\
\text { filter size computed from } \Delta_{f}=V^{1 / 3} . \sigma_{k} \text { is } \\
\text { 1.0. }\end{array}$ \\
\hline
\end{tabular}

TABLE 2: CFD model description.

\begin{tabular}{lc}
\hline Analysis type & Transient state \\
\hline Subgrid-scale models & SL, DSL, WALE, WMLES, \\
Pressure-velocity coupling & WMLES $S$ - $\Omega$, and KET \\
Transient formulation & SIMPLEC \\
Momentum & Bounded central differencing \\
Pump status & Varied from $200 \mathrm{rpm}$ to \\
Turbine status & $600 \mathrm{rpm}$ \\
Viscosity and density & Stationary \\
\hline
\end{tabular}

was larger than that in other models. SL, DSL, and KET could simulate a relatively small Re values in the near-wall region and a relatively moderate Re values in the mainstream.
3.2. Vorticity Distribution. Vorticity was a physical parameter to indicate the vortex motion and represent the energy transportation process. The generation of vortex was caused by the velocity gradient in the flow field. Its development, diffusion, and decline were caused by the viscous interaction in the flow field. The value of vorticity was two times that of the rigid rotation angular velocity of fluid micelle.

Figure 3 shows the vorticity distribution patterns of the mid chord R120 in the flow field obtained with all subgridscale models. The large vorticity values were concentrated in the near-wall area in the turbine blade and the low vorticity values were concentrated in middle cascade area, especially in the pump wheel. The vorticity ranged from 0 to $46001 / \mathrm{s}^{2}$. In the braking process, the pump impeller stirred oil to convert the mechanical energy into kinetic energy of working oil. Then the oil flowed into the turbines and impacted the turbine blades, thus producing strong relative effects between the oil and the turbine blade surface. Then a large number of 


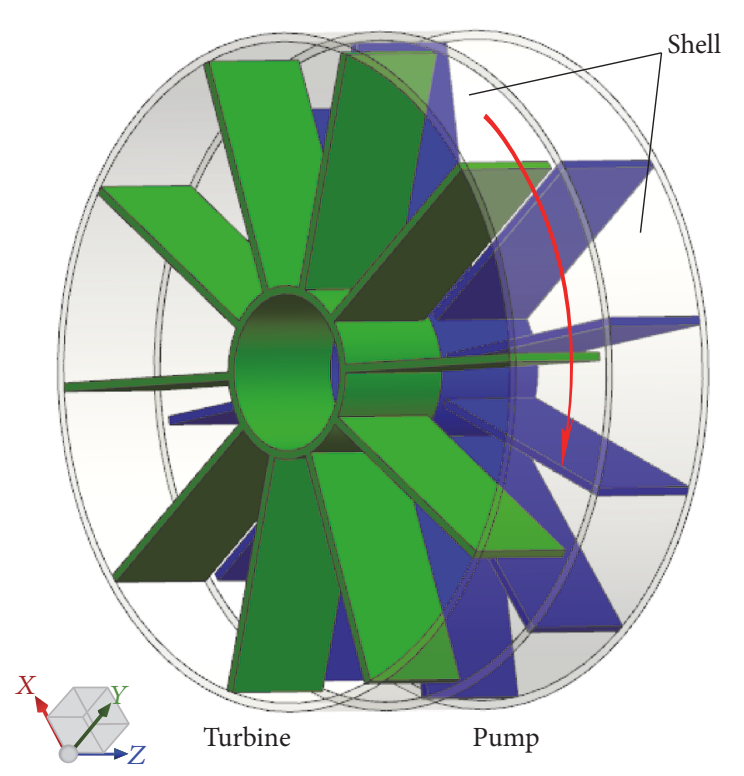

(a)

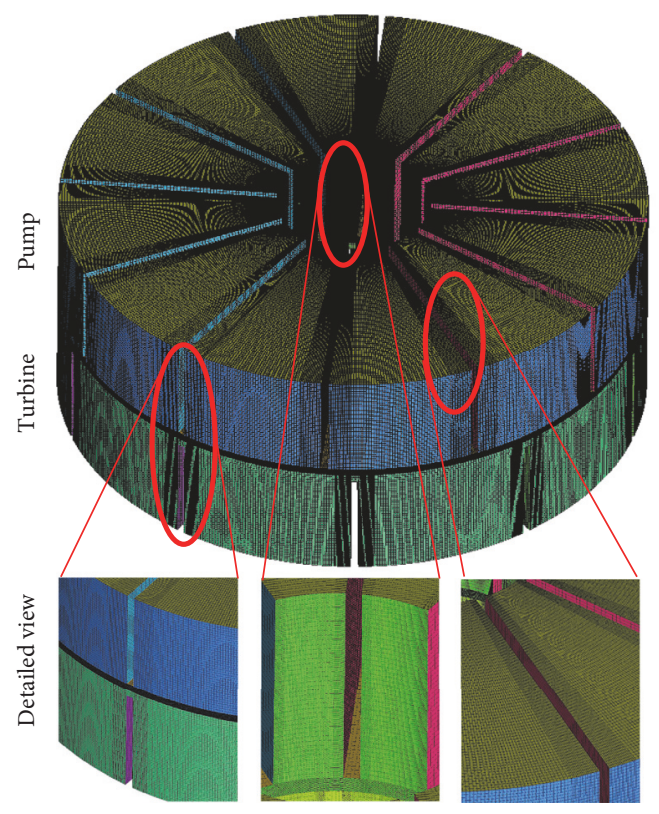

(b)

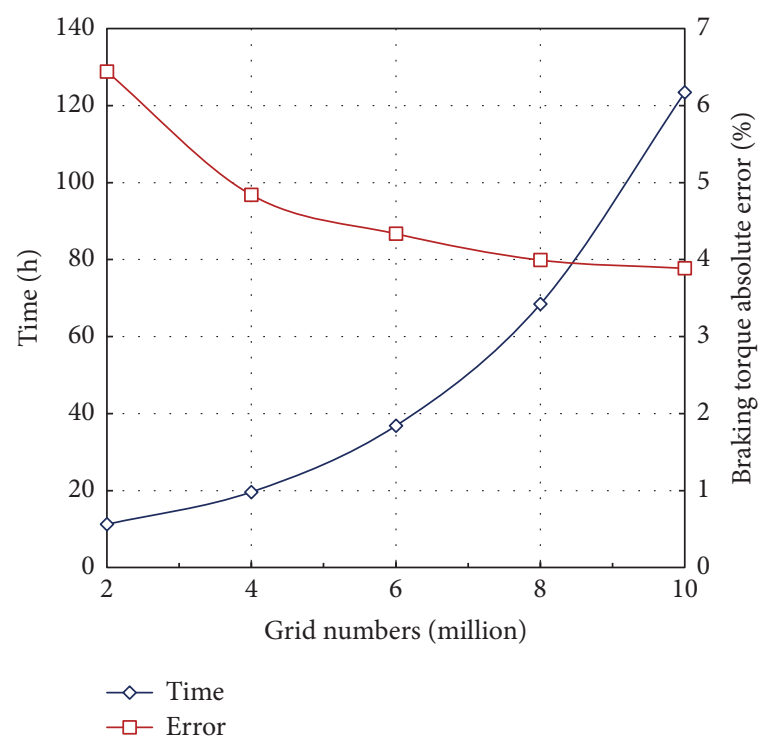

(c)

FIGURE 1: Hydraulic coupling. (a) 3D model; (b) grid layout; (c) grid independence test conditions.

vortexes were generated. In the SL model, vortexes formed inside the turbine were scattered and the vortex distribution near the wall was thin. In DSL, the adopted dynamic change $C_{d}$ led to ample and continuous vorticity in turbine, which could improve the capture ability of vortex effectively. High vorticity value distribution of WALE was scattered and the predicted value of WALE was larger than that of other models. The remaining three SGS models could well capture the abundant structure of vorticity distribution, and the vorticity in the turbine also showed the continuous distribution. In conclusion, in the large eddy simulation, WMLES, WMLES $S-\Omega$, and KET models showed more advantages in analyzing the vorticity distribution in the flow field and the distribution structures were abundant and continuous.

3.3. Changes in Rothalpy. Figure 4 describes the changes of Rothalpy from the inlet to the outlet on the nodal surface of turbine under different subgrid models. The nondimensional distance $x$ could be defined as

$$
x=\frac{S}{L} .
$$

$L$ was the length of flow streamline on turbine's surface. If a point cut the flow streamline into two segments, then 


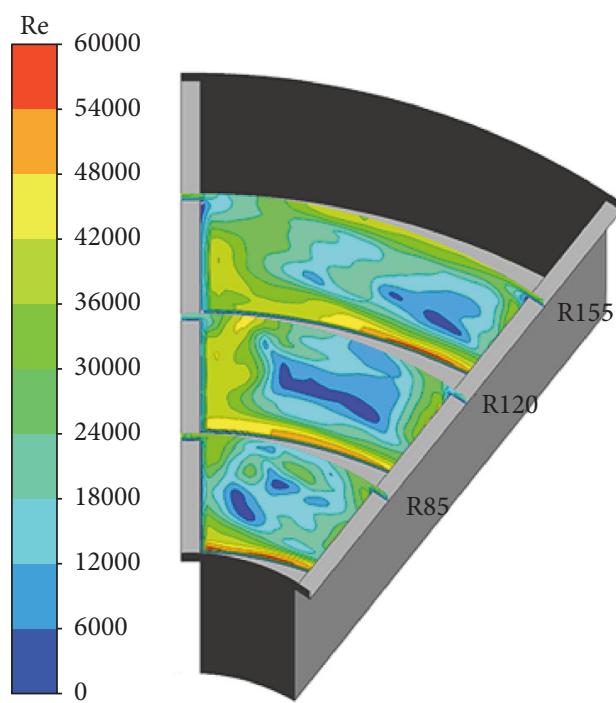

SL

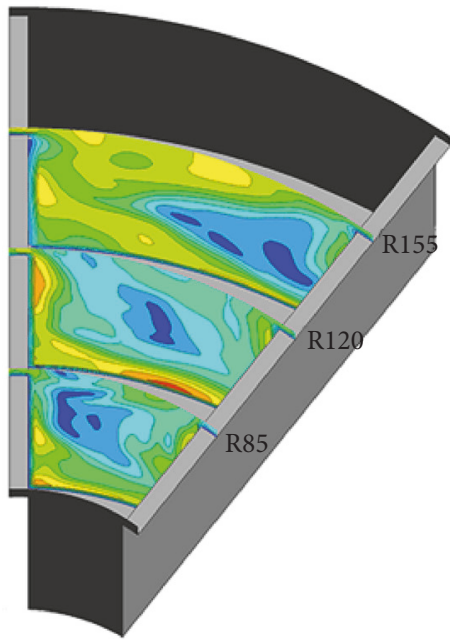

WMLES

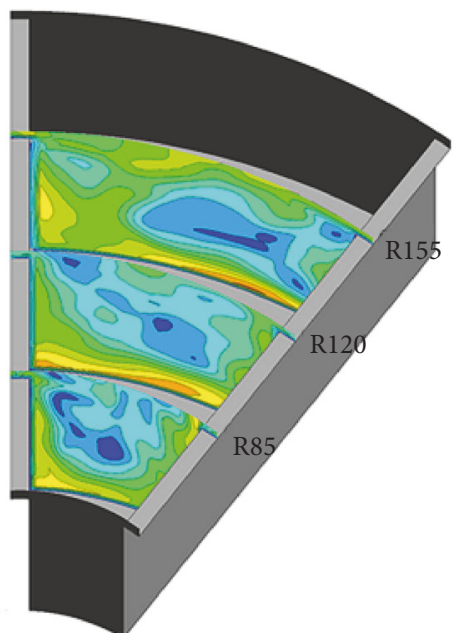

DSL

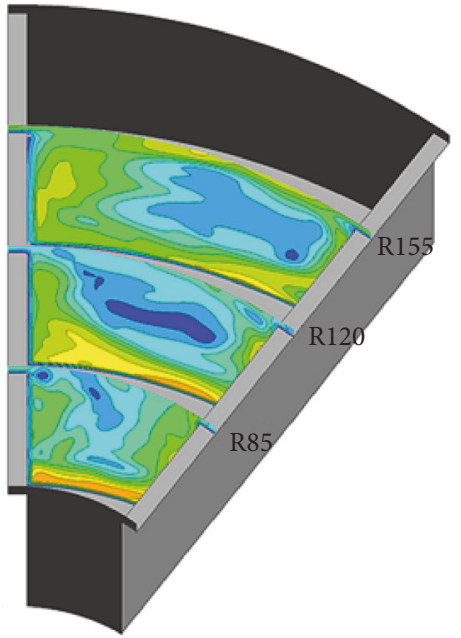

WMLES $S-\Omega$

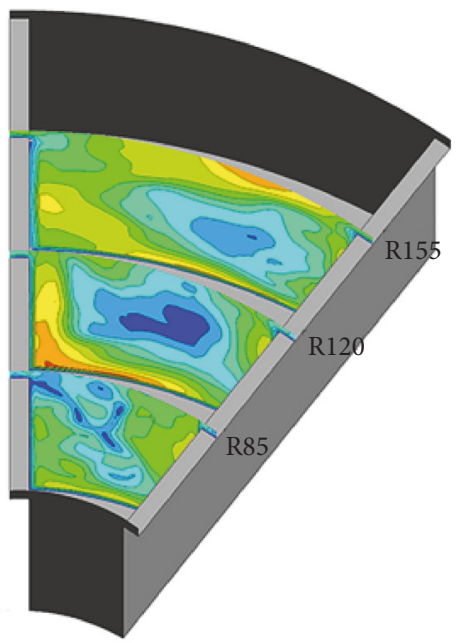

WALE

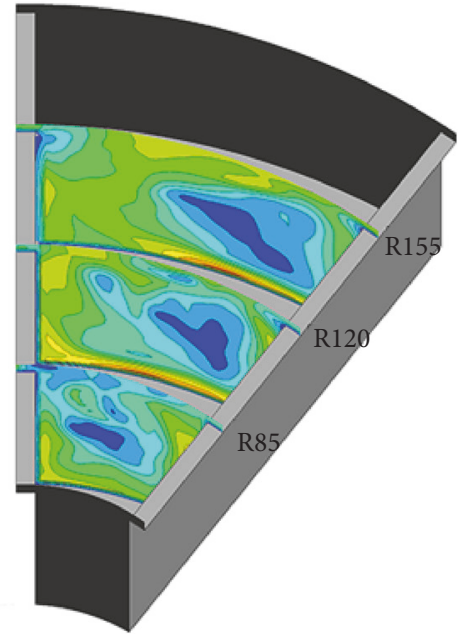

KET

Figure 2: Re distribution on radial sections.

the part approaching the turbine's inlet was marked as part one. $S$ was the length of part one. "0" and " 1 " represented, respectively, the locations of inlet and outlet of turbine. The $x$ coordinates of $\mathrm{A}, \mathrm{B}, \mathrm{C}$, and $\mathrm{D}$ point were $0.04,0.60,0.73$, and 0.96 , respectively. "A" and "B" in Figure 4 represent the leading and trailing edges, respectively. On the whole, Rothalpy value was decreased gradually with the increase in dimensionless distance, suggesting that the hydraulic loss was generated when oil impacted the turbine blades. In Figure 4, Point A shows that the oil flowed from the nonblade area into the blade edge. Rothalpy was reduced because the leading edge of blades hampered the working oil from flowing into the turbine. Correspondingly, hydraulic loss behind Point B referred to the diffusion loss generated when oil flowed into the nonblade area. Additionally, the regional largest changes of Rothalpy appeared in Points C and D.
Among various models, the SL model allowed the largest change of Rothalpy, 31.4\%; WMLES and WALE provided almost the consistent change, 28.7\%; WMLES $S$ - $\Omega$ gave the change of $26.3 \%$. The changes in the results of DSL and KET were the smallest, $24.9 \%$. The phenomenon could be interpreted as follows: Rothalpy reflected the interaction strength between oil and the blades to a certain extent, indicating that the more intense interactions and viscosity dissipation led to the more obvious Rothalpy changes and the larger brake torque. These tendencies were consistent with that of the predicted braking torque obtained with various models.

\section{Flow Mechanism Analysis}

Q-Criterion was introduced to capture the vortex structure in the flow field. The Q-Criterion was defined as the second 

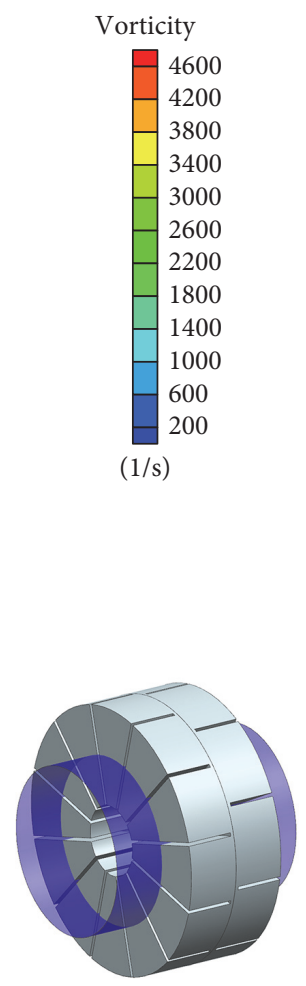

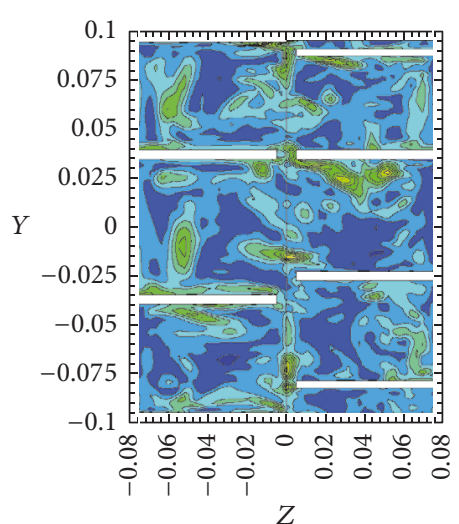

SL

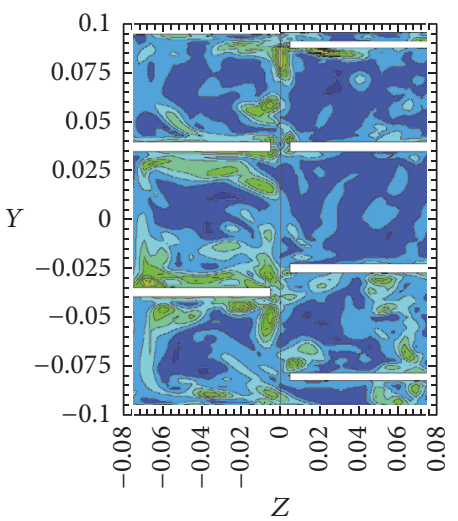

WMLES

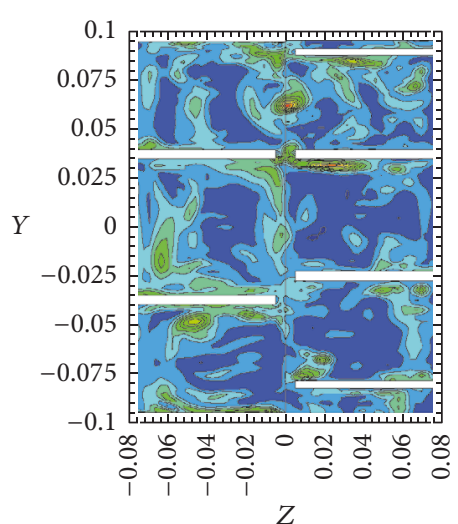

DSL

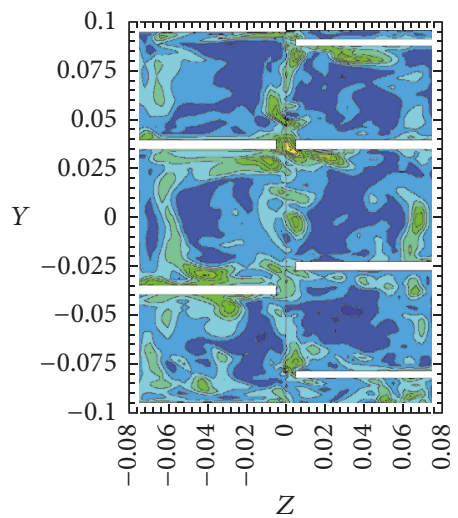

WMLES $S-\Omega$

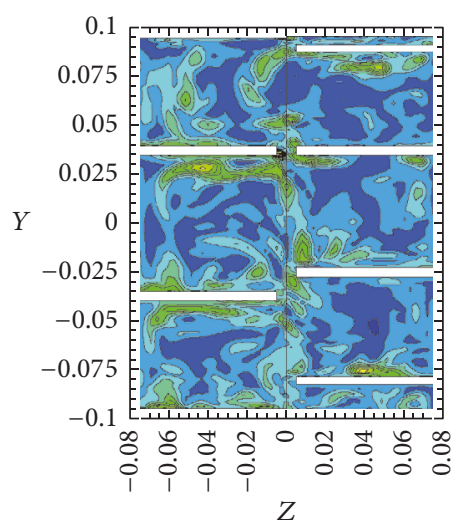

WALE

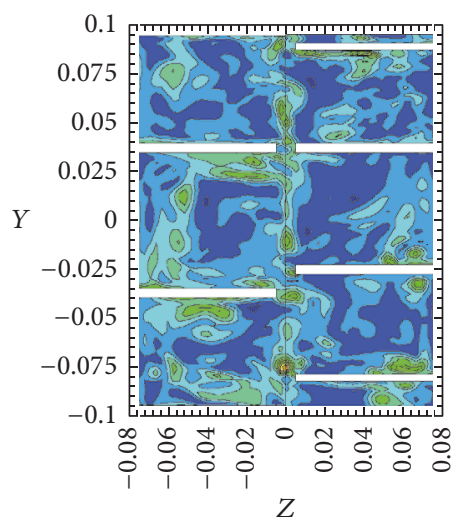

KET

FIgURE 3: Vorticity distribution for different subgrid models.

invariant of the velocity gradient tensor. Under the hypothesis of incompressible flow, $Q$ could be written as

$$
Q=\frac{1}{2}\left(\|\Omega\|^{2}-\|S\|^{2}\right) \text {. }
$$

||| represented the 2-norm of tensor, where $S$ and $\Omega$ were the symmetric and antisymmetric portions of the velocity gradient tensor, respectively. The $Q$ value showed the degree that the rotational rate $\|\Omega\|^{2}$ overcame the strain rate $\|S\|^{2}$; therefore, positive $Q$ value corresponded to dominant eddy.

As a comparison, the vortex structures of the SAS and RKE, and SSTKW models were also calculated and presented to highlight the ability of capturing the vortex structures for those SGS models. Figure 5 reveals the ability to capture the vortex structures for SGS models within the turbine under $Q=8.7 \times 10^{5} 1 / \mathrm{s}^{2}$. Because the effect of all turbulent eddies was mimicked in the turbulence model of RANS, the effect of most turbulence eddies was directly simulated in the LES. It was evident that those SGS captured more vortex structures than SAS and RANS. Meanwhile, SAS and SSTKW provided the higher value of eddy viscosity ratio than SGS. Among the SGS models, DSL and WMLES $S-\Omega$ captured the most abundant vortex structures, followed by KET. SL provided a relatively larger eddy viscosity compared to other SGS models, followed by WMLES and WALE. WMLES $S-\Omega$ provided the smallest eddy viscosity. As a result, DSL and

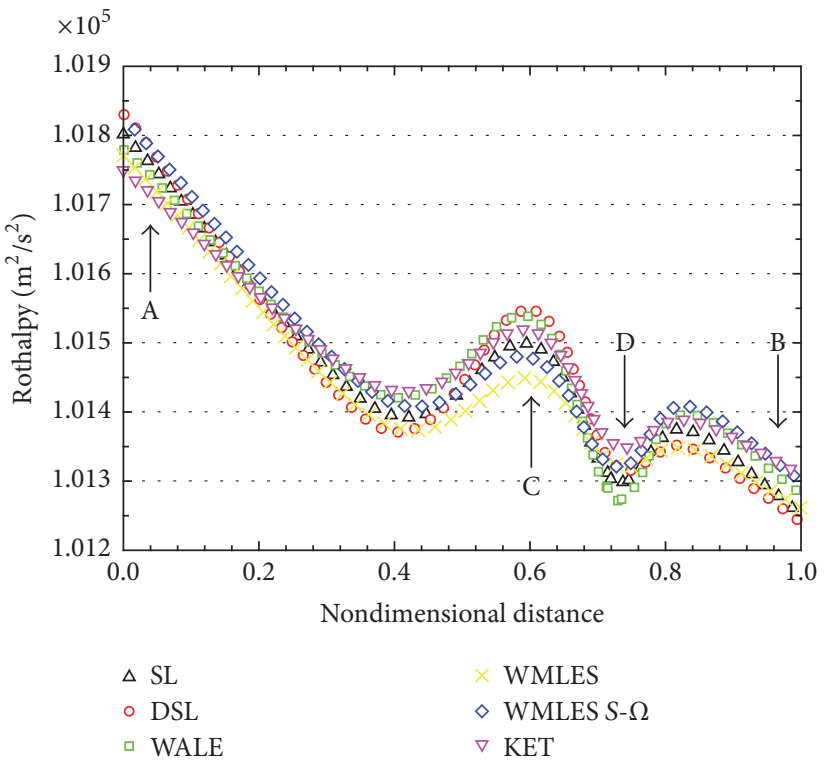

FIGURE 4: Changes in Rothalpy for different subgrid models.

KET captured abundant vortex structures and provided a relatively moderate eddy viscosity. 


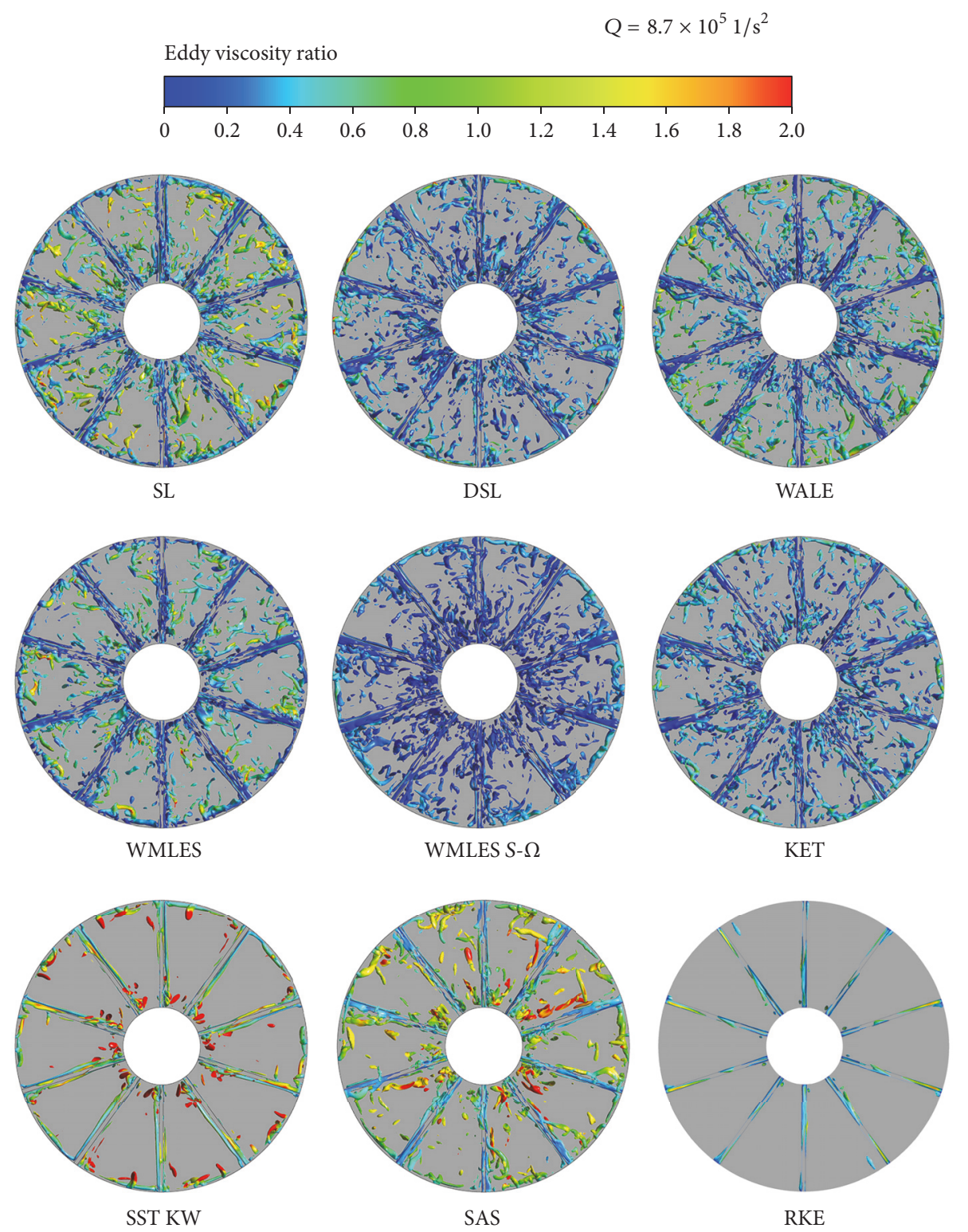

FIgURE 5: Vortex structures within turbine.

Figure 6 shows the vortex structures as a whole from front view of pump. The vortexes were concentrated in the region which was from the hub to the one-third blade height, where the kinetic energy of fluid transmission took place under $600 \mathrm{rpm}$. If the engineer wanted to advance the performance through flow field control, boundary control, just like the work in $[5,6]$, the position obtained could be significant, which inspired directly and avoided the attempts. The models all gave rich vortexes and were hardly told the differences through qualitative analysis, except SSTKW, RKE, and SAS.

Figure 7 shows the vortex structures in interior of the pump and turbine. Thus we found that there were several eddy structures along the wall of flow field, and it was diverse under different models. Fluid impacted on the impeller directly, so there was a linear flow eddy in the blade of the pump and turbine. Linear flow was divided into two sections, namely, the straight flow near the upper portion of the outer ring and the high-speed linear flow in the foot of the inner ring.

\section{Performance Prediction and Verification}

The comparison between the experimental and simulated results is plotted in Figure 8(a). To facilitate the comparison of the prediction ability of braking performance of hydraulic coupling among various models, we introduced the braking torque error defined as

$$
E_{S}=\frac{\left|T_{E}-T_{S}\right|}{T_{E}} \times 100 \%,
$$

where $T_{E}$ was the experimental torque value; $T_{S}$ represented the torque value of simulation prediction; $E_{S}$ was the absolute 


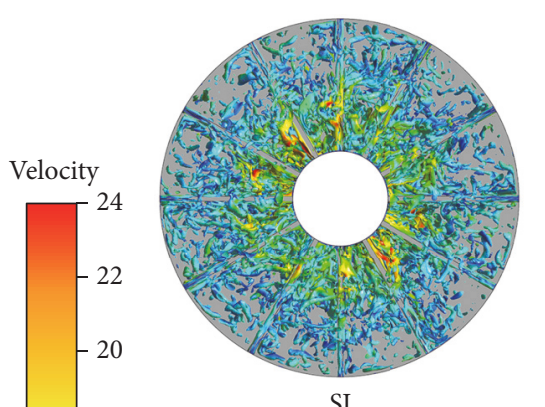

SL

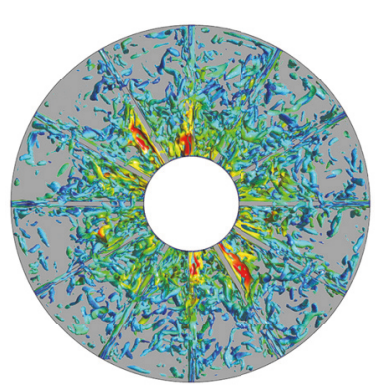

WMLES

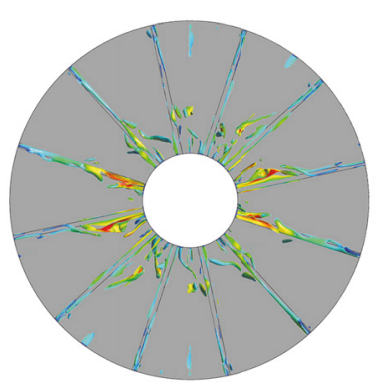

SST KW

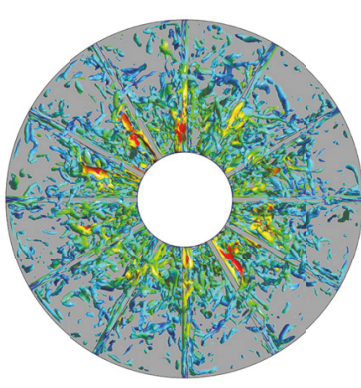

DSL

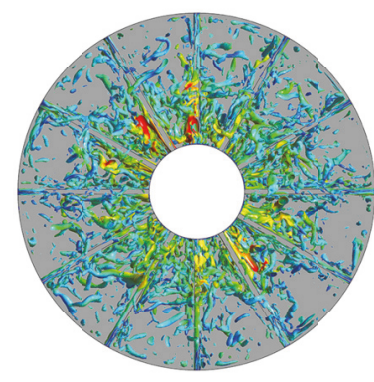

WMLES $S-\Omega$

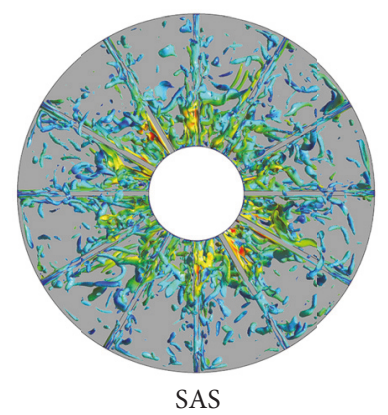

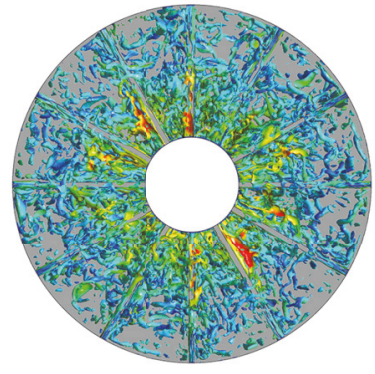

WALE

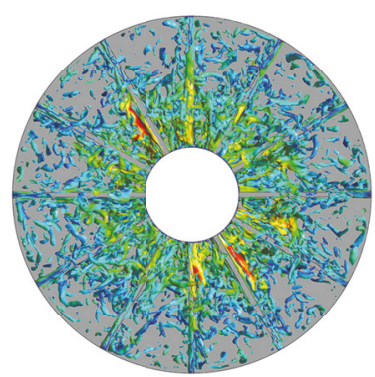

KET

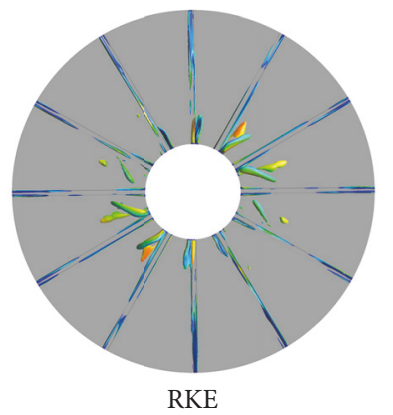

FIGURE 6: Vortex structures in whole flow channel.

error of braking torque prediction. Figure 8 (b) shows the prediction errors of each subgrid-scale model. The errors of DSL and KET were less than 5\% under low speed, and the DSL was lower, but the error of SL was the highest. Except the SL, all the models provided the errors below $4 \%$ under high speed. Moreover, with the increase in the speed, except the SL, all the models allowed the decreasing errors below 5\%. The results were compared with the previous researchers: in the study of Sun et al. [22], with the standard $k-\varepsilon$ model, the error was within $7.5 \% \sim 15 \%$. Fortunately, a lower error was acquired in He et al's research [23], and the error was close to but more than $6 \%$ under the pump rotational speed of $600 \mathrm{rpm}$ by using standard $k-\varepsilon$ model. However, in the research of Song et al. [24], the error was just less than $10 \%$ with the realizable $k-\varepsilon$ model. The results of these explained that the error of subgrid model was more accurate than the former researcher's model.

Therefore, at the low speed, due to the influences of other factors in the flow field, the weak centrifugal force, and the insufficient flow, the predicted values were relatively large. With the increase in the rotating speed, the proportion of the centrifugal force increased and the flow developed fully. Therefore, other factors could be ignored and the prediction condition was closer to the ideal condition. In conclusion, at low speed, the higher order of DSL and KET subgridscale models was more advantageous to the performance prediction. Except the SL one, the other models showed the consistent prediction abilities for the performance at high speed.

Furthermore, the SAS and RKE, and SSTKW models were also calculated. SAS was a new class of URANS models which had been developed that could provide a LES-like behavior in unsteady flow regions. Meanwhile, they were more compatible with LES formulations than standard RANS models. RKE and SSTKW were typical RANS models. As shown in Figure 8(b), the error of those three is larger than that of the LES subgrid-scale models. The results showed that prediction accuracy of LES was higher than RANS and SAS.

Within the boundary layer, the velocity near the wall compared to the free flow speed would be decreased to zero quickly. Therefore, the velocity gradient was large. Especially in the viscous sublayer, the viscosity played the main role. With the increase of the distance to the wall, the velocity gradient decreased. Meanwhile, the viscosity effect reduced, but the inertia effect increased, and the flow finally developed into the full turbulence. Therefore, the interpretation of boundary layer transition was an important standard of a 


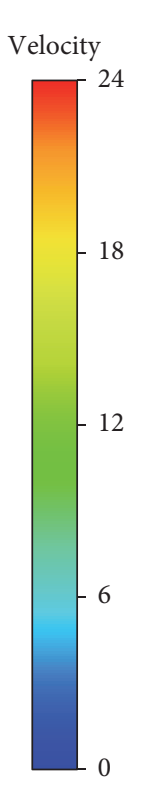

$(\mathrm{m} / \mathrm{s})$
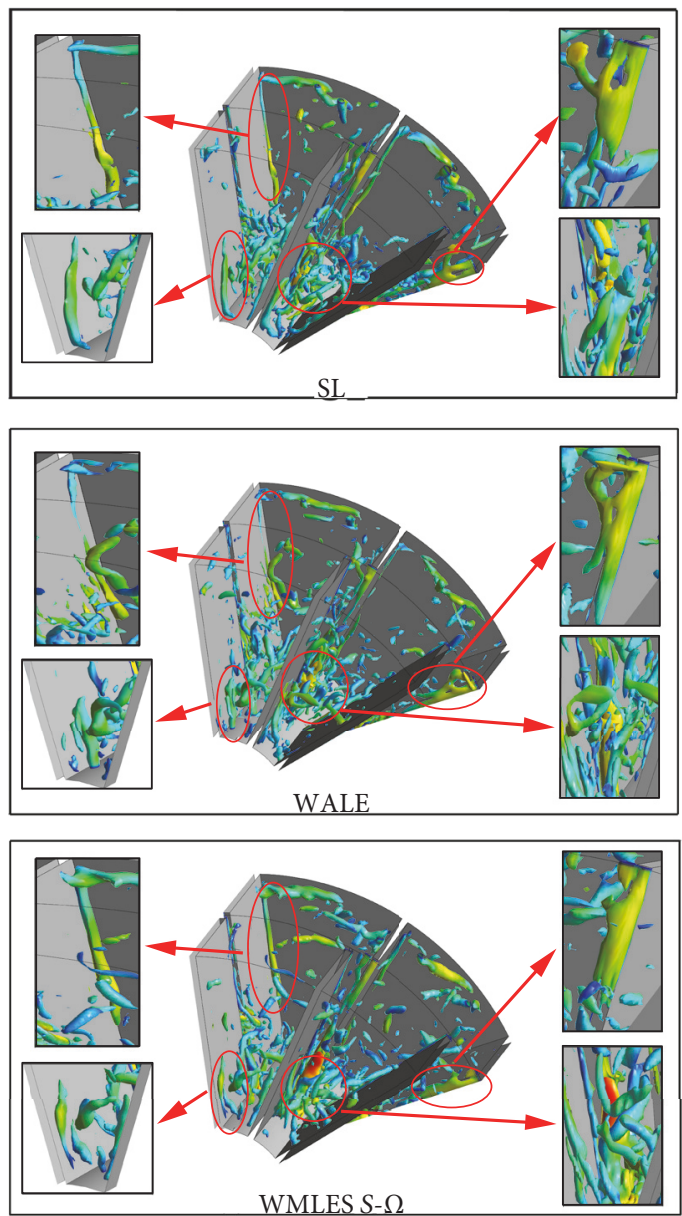
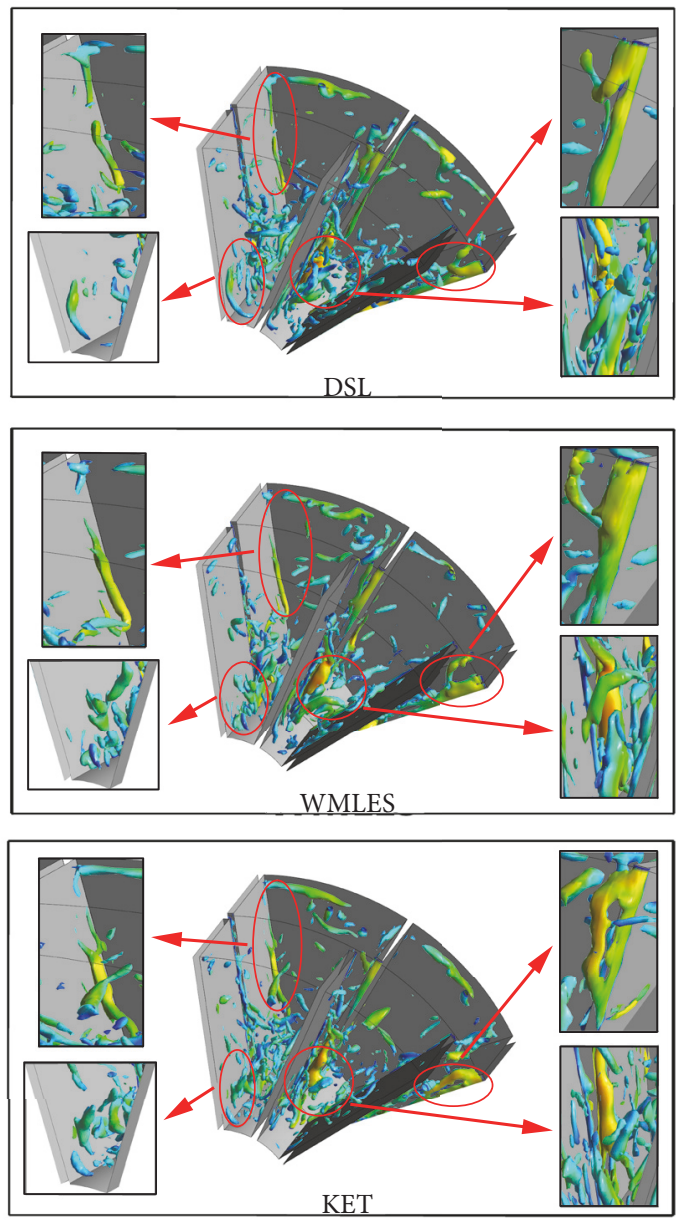

FIGURE 7: The vortex structures and flow field in the interior.

turbulence model. The reasons for improvement were mainly including their capacity of treatment for near-wall with fine grid. The near-wall treatments consisted of applying wall functions and resolving the viscous sublayer. Within the boundary layers, the most important region was the viscous sublayer, immediately adjacent to the wall and the log law region, slightly further away from the wall. Usually the nearwall mesh should be fine enough to be able to resolve the viscous sublayer, typically with the first near-wall node placed at. In this case, the SGS models could resolve effectively the viscous sublayer.

Figure 9 shows the analytical ability to the boundary layer of various grids models in the turbine pressure surface, where the RANS and SAS fail to get the data. DSL, WMLES, WMLES $S-\Omega$, and KET could solve the viscous sublayer well. In addition, the interpretations of DSL and KET in the viscous sublayer were richer, especially at $y^{+} \approx 1$. The results indicated that the dynamic stress solution of subgrid-scale model in the viscous sublayer was more advantageous. The locations of the transition layer in six models were similar. In other locations, according to the comparison results of the $u^{+}$value under the same value of $y^{+}$of various models, the WMLES allowed the largest predicted value and the SL allowed the smallest predicted value. With the increase of $y^{+}$ value, the $u^{+}$values of six models tended to be consistent because the $N-S$ equations were adopted to solve the transient in the mainstream zone.

In conclusion, DSL and KET performed better than other models in solving the boundary layer. Moreover, DSL and KET allowed the better predicted value of the braking torque of the hydraulic coupling under low speed conditions.

\section{Conclusions}

In order to study different ability of six subgrid-scale models to predict the flow field and performance of a fluid coupling, some studies, such as Re distribution, vorticity distribution, changes in Rothalpy, flow mechanism analysis, the error of braking torque prediction, and analytical ability to the boundary layer, had been conducted. Some conclusions had been drawn.

In the descriptions of the Re distribution, SL, DSL, and KET could simulate a relatively small Re values in the nearwall region and a relatively moderate Re values in the mainstream. In conclusion, in the large eddy simulation, WMLES, WMLES $S-\Omega$, and KET models showed more advantages in analyzing the vorticity distribution in the flow field and the distribution structures were abundant and continuous. 


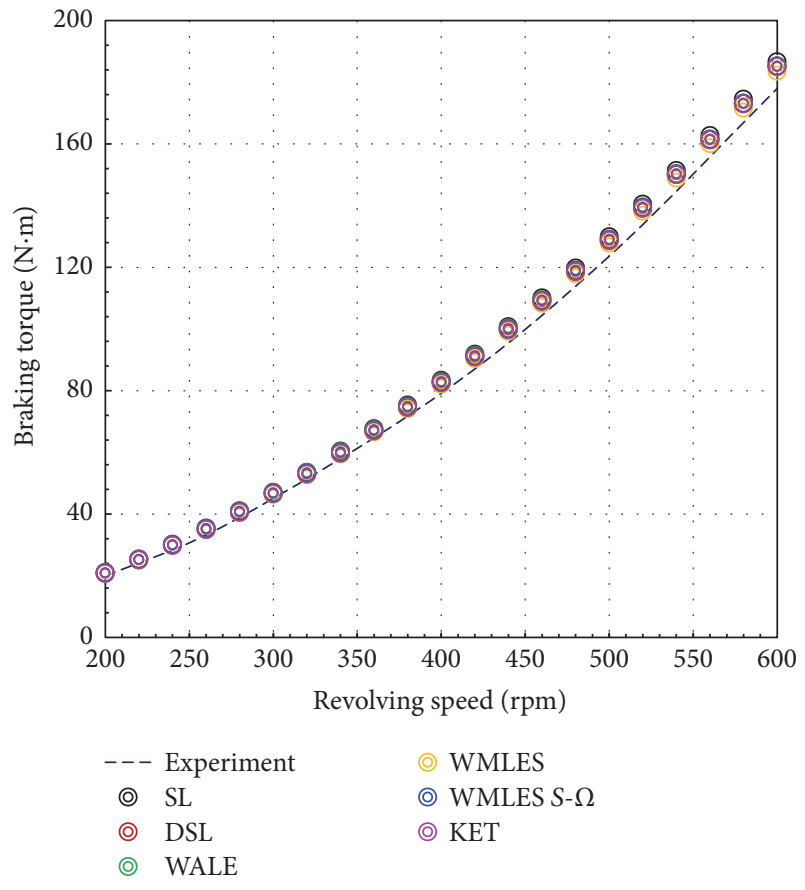

(a)

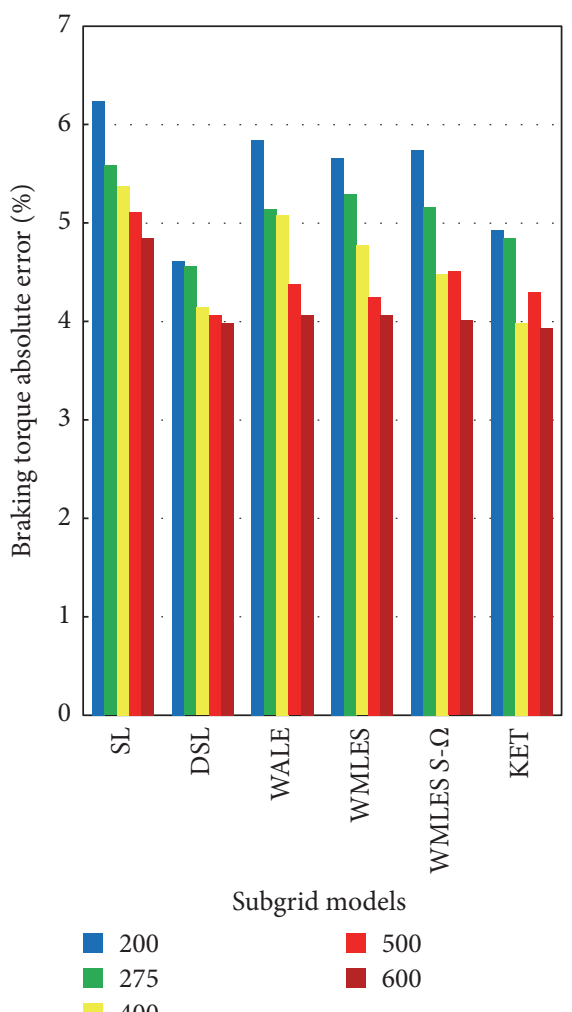

(b)

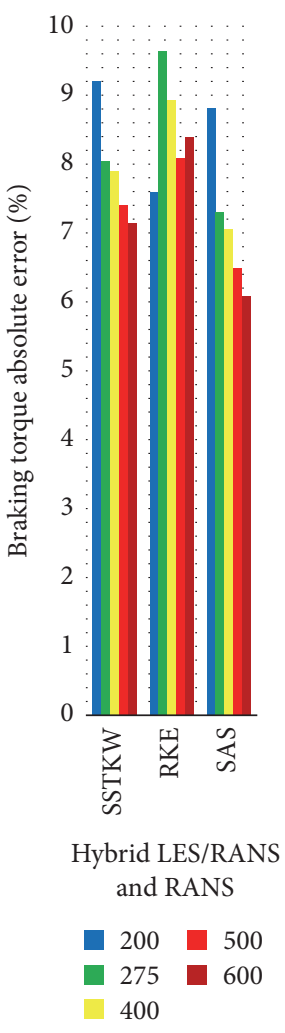

FIGURE 8: Braking torque calculated results. (a) Experimental and calculated results. (b) Braking torque absolute error.

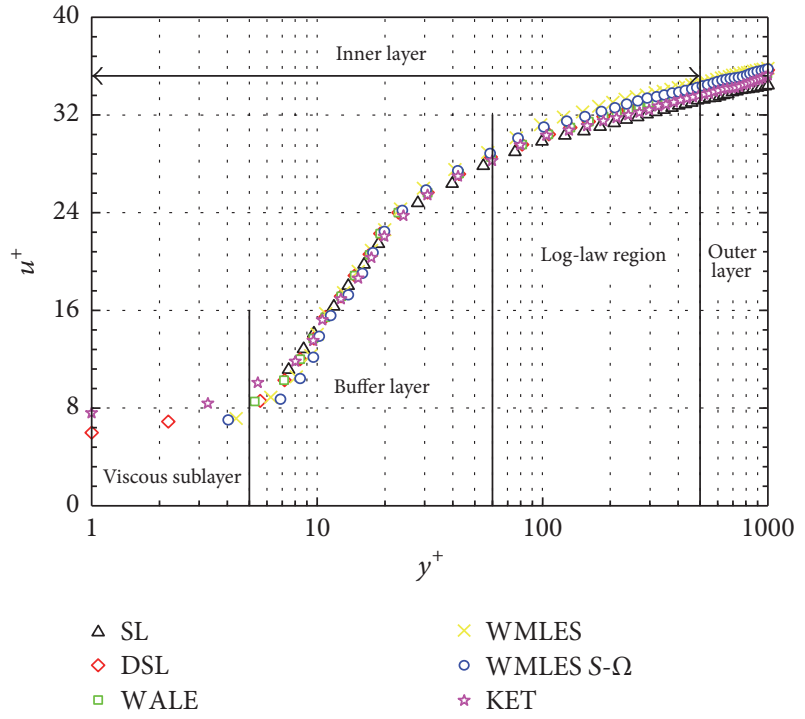

FIGURE 9: Boundary layer profiles near the pressure surface for turbine.

To some degree, Rothalpy changes reflected the strength of the interaction between oil and blade surface. The more intense interaction and the more viscosity dissipation meant the more obvious Rothalpy changes. Correspondingly, brake torque also was greater.
As we all know, the effect of all turbulent eddies was mimicked in the turbulence model of RANS, but the effect of most turbulence eddies was directly simulated in the LES. As a result, DSL and KET captured abundant vortex structures and provided a relatively moderate eddy viscosity within turbine. When the vortex structures as a whole were observed from front view of pump, the vortexes were concentrated in the region which was from the hub to the one-third blade height, where the kinetic energy of fluid transmission took place under $600 \mathrm{rpm}$. The models all gave rich vortexes and were hardly told the differences through qualitative analysis, except SSTKW, RS, and SAS. Because fluid impacted on the impeller directly, a linear flow eddy appeared in the blade of the pump and turbine.

In the prediction of braking torque, DSL and KET showed the smallest errors (less than 5\%) at low speed, while the errors of other models were more than 5.6\%. With the increase in rotational speed, the prediction errors of all the subgrid-scale models except SL model gradually maintained at $4 \%$. DSL, WMLES, WMLES $S-\Omega$, and KET may solve the viscous sublayer well. In addition, the interpretations of DSL and KET in the viscous sublayer were richer, especially at $y^{+} \approx 1$. It meant that DSL and KET performed better than other models in solving the boundary layer. It led to the smallest errors showed by DSL and KET. Moreover, RKE, SSTKW, and SAS were also calculated. These results showed that SGS models were more accurate by contrast. Moreover, the predicted results of DSL and KET were far more accurate 
than the former study. Therefore, in the prediction of braking torque, we could choose DSL or KET subgrid-scale model to obtain the predicted results.

In conclusion, the six SGS models, especially DSL and KET, were applicable to obtain the more accurate predicted results and depict the flow field. The study not only provided the detailed study method for the application of large eddy simulation in hydraulic coupling, but also proposed many opinions on the model selection.

\section{Conflicts of Interest}

The authors declared that there were no conflicts of interest regarding the publication of this paper.

\section{Acknowledgments}

This work was also supported by the National Natural Science Foundation of China (Grant no. 51675219).

\section{References}

[1] C. N. McKinnon, D. Brennen, and C. E. Brennen, "Hydraulic analysis of a reversible fluid coupling," Journal of Fluids Engineering, Transactions of the ASME, vol. 123, no. 2, pp. 249-255, 2001.

[2] U. Hampel, D. Hoppe, K.-H. Diele et al., "Application of gamma tomography to the measurement of fluid distributions in a hydrodynamic coupling," Flow Measurement and Instrumentation, vol. 16, no. 2-3, pp. 85-90, 2005.

[3] U. Hampel, D. Hoppe, A. Bieberle et al., "Measurement of fluid distributions in a rotating fluid coupling using high resolution gamma ray tomography," Journal of Fluids Engineering, Transactions of the ASME, vol. 130, no. 9, pp. 0914021-0914028, 2008.

[4] L. Bai, M. Fiebig, and N. K. Mitra, "Numerical analysis of turbulent flow in fluid couplings," Journal of Fluids Engineering, Transactions of the ASME, vol. 119, no. 3, pp. 569-576, 1997.

[5] H. Huitenga and N. K. Mitra, "Improving startup behavior of fluid couplings through modification of runner geometry: part I-fluid flow analysis and proposed improvement," Journal of Fluids Engineering, Transactions of the ASME, vol. 122, no. 4, pp. 683-688, 2000.

[6] H. Huitenga and N. K. Mitra, "Improving startup behavior of fluid couplings through modification of runner geometry: part II-modification of runner geometry and its effects on the operation characteristics," Journal of Fluids Engineering, Transactions of the ASME, vol. 122, no. 4, pp. 689-693, 2000.

[7] Y. Luo, L. H. Feng, S. H. Liu, T. J. Chen, and H. G. Fan, "Numerical comparisons of the performance of a hydraulic coupling with different pump rotational speeds," in Proceedings of the 6th International Conference on Pumps and Fans with Compressors and Wind Turbines (ICPF '13), vol. 52, no. 7, IOP Publishing, September 2013.

[8] Y. Luo, Z. G. Zuo, S. H. Liu, H. G. Fan, and W. L. Zhuge, "Numerical simulation of the two-phase flows in a hydraulic coupling by solving VOF model," in Proceedings of the 6th International Conference on Pumps and Fans with Compressors and Wind Turbines (ICPF '13), vol. 52, no. 7, IOP Publishing, September 2013.

[9] N. Hur, M. Kwak, W. J. Lee, M. Moshfeghi, C.-S. Chang, and N.-W. Kang, "Unsteady flow analysis of a two-phase hydraulic coupling," in Proceedings of the International Conference of Numerical Analysis and Applied Mathematics 2015 (ICNAAM '15), vol. 1738, no. 1, AIP Publishing, September 2015.

[10] N. Gourdain, F. Sicot, F. Duchaine, and L. Gicquel, "Large eddy simulation of flows in industrial compressors: a path from 2015 to 2035," Philosophical Transactions of the Royal Society A: Mathematical, Physical and Engineering Sciences, vol. 372, no. 2022, Article ID 20130323, 2014.

[11] F. Duchaine, N. Maheu, V. Moureau, G. Balarac, and S. Moreau, "Large-Eddy simulation and conjugate heat transfer around a low-mach turbine blade," Journal of Turbomachinery, vol. 136, no. 5, Article ID 051015, 2013.

[12] C. Trivedi, M. J. Cervantes, and O. G. Dahlhaug, "Experimental and numerical studies of a high-head Francis turbine: a review of the Francis-99 test case," Energies, vol. 9, no. 2, pp. 1-24, 2016.

[13] B. Wu, "Large eddy simulation of mechanical mixing in anaerobic digesters," Biotechnology and Bioengineering, vol. 109, no. 3, pp. 804-812, 2012.

[14] T. Lu, Y. Wang, and P. Wang, "Large eddy simulation with three kinds of sub-grid scale model on temperature fluctuation of hot and cold fluids mixing in a tee," Applied Mechanics and Materials, vol. 152-154, pp. 1307-1312, 2012.

[15] M. Jahoda, M. Moštěk, A. Kukuková, and V. Machoň, "CFD modelling of liquid homogenization in stirred tanks with one and two impellers using large eddy simulation," Chemical Engineering Research and Design, vol. 85, no. 5 A, pp. 616-625, 2007.

[16] Z. Li, G. Song, Y. Bao, and Z. Gao, "Stereo-PIV experiments and large eddy simulations of flow fields in stirred tanks with Rushton and curved-Blade turbines," AIChE Journal, vol. 59, no. 10, pp. 3986-4003, 2013.

[17] G. Lodato, L. Vervisch, and P. Domingo, "A compressible walladapting similarity mixed model for large-eddy simulation of the impinging round jet," Physics of Fluids, vol. 21, no. 3, Article ID 035102, 2009.

[18] B. Ji, X.-W. Luo, X.-X. Peng, and Y.-L. Wu, “Three-dimensional large eddy simulation and vorticity analysis of unsteady cavitating flow around a twisted hydrofoil," Journal of Hydrodynamics, vol. 25 , no. 4 , pp. 510-519, 2013.

[19] R. Menter Florian, Turbulence Modeling for Engineering Flows, Ansys Inc, 2011.

[20] S. Patil and D. Tafti, "Large-eddy simulation with zonal near wall treatment of flow and heat transfer in a ribbed duct for the internal cooling of turbine blades," Journal of Turbomachinery, vol. 135, no. 3, Article ID 031006, 2013.

[21] M. Ricci, L. Patruno, S. de Miranda, and F. Ubertini, "Effects of low incoming turbulence on the flow around a 5:1 rectangular cylinder at non-null-attack angle," Mathematical Problems in Engineering, vol. 2016, Article ID 2302340, 12 pages, 2016.

[22] Z. Sun, J. Chew, N. Fomison, and D. Edwards, "Analysis of fluid flow and heat transfer in industrial fluid couplings," Proceedings of the Institution of Mechanical Engineers, Part C: Journal of Mechanical Engineering Science, vol. 223, no. 9, pp. 2049-2062, 2009.

[23] Y. He, W. Ma, and C. Liu, "Numerical simulation and characteristic calculation of hydrodynamic coupling," Transactions of the Chinese Society of Agricultural Machinery, vol. 40, no. 5, pp. 24-28, 2009.

[24] B. Song, J. Lv, S. Guo, X. Zhang, and F. Kong, "Simulation and characteristic analysis on flow field of fluid couplings during braking," Machine Design \& Research, vol. 27, no. 1, pp. 26-30, 2011. 


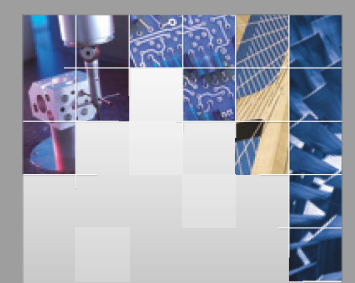

\section{Enfincering}
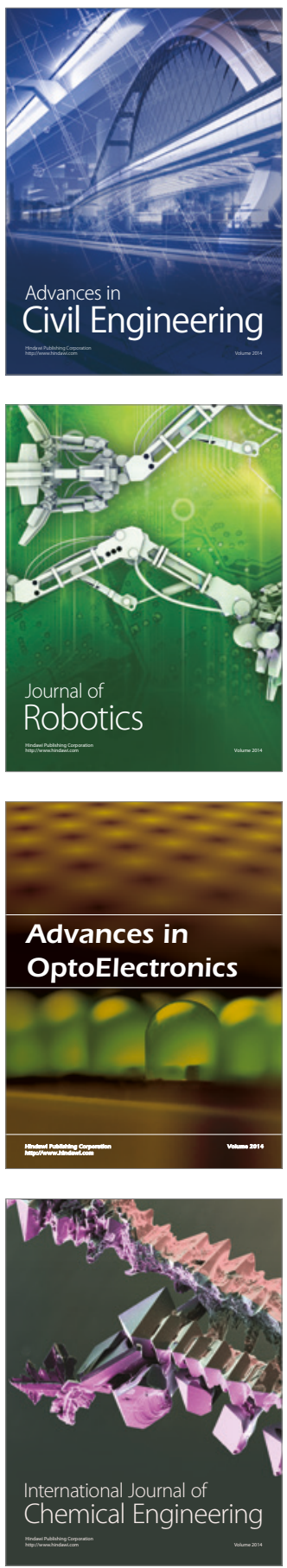

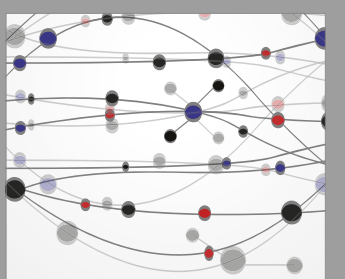

The Scientific World Journal

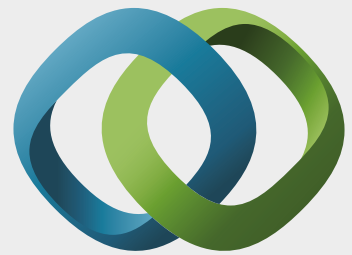

\section{Hindawi}

Submit your manuscripts at

https://www.hindawi.com
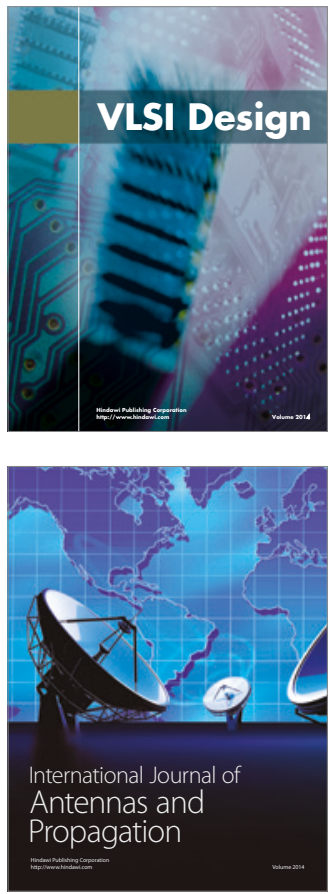

\section{Rotating}

Machinery
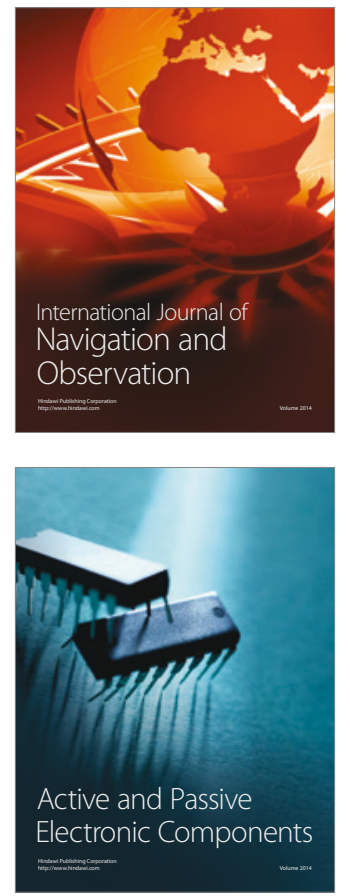
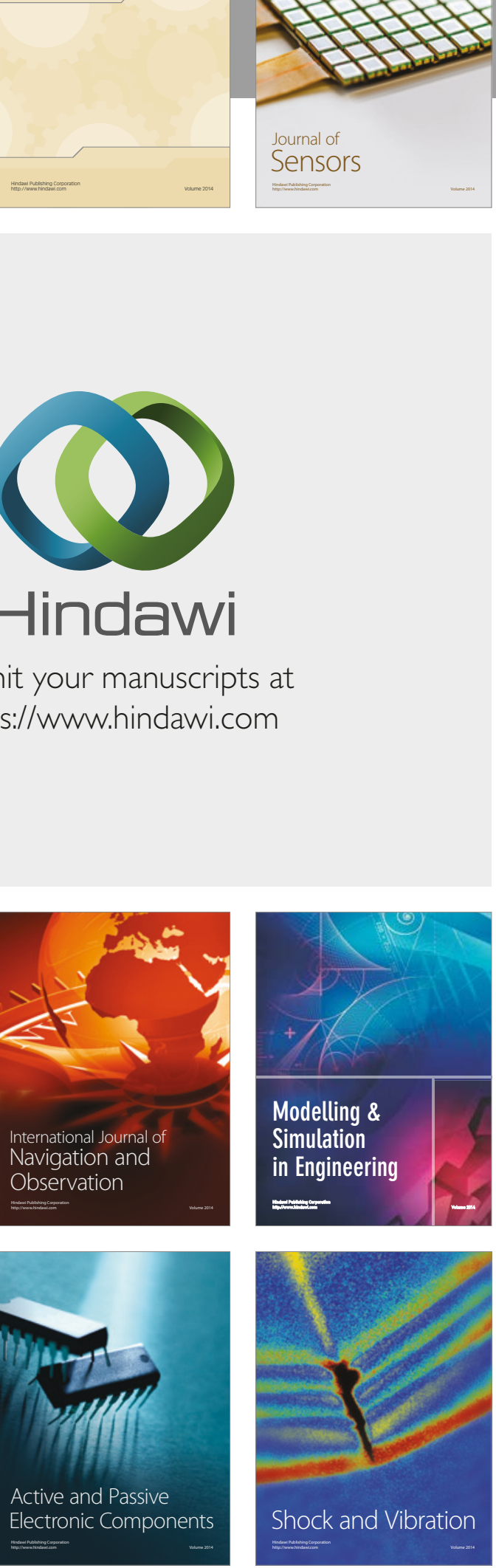
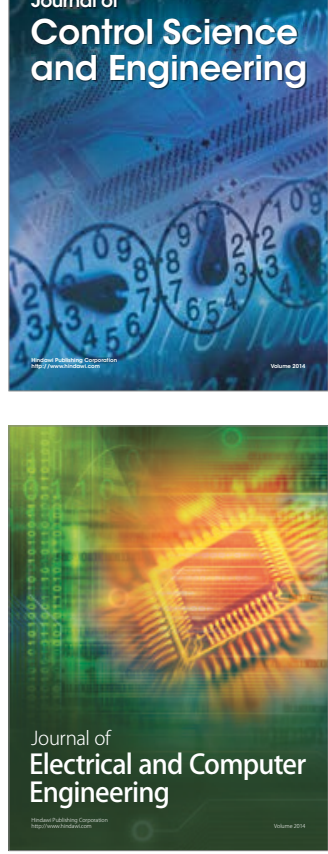

Distributed

Journal of

Control Science

and Engineering
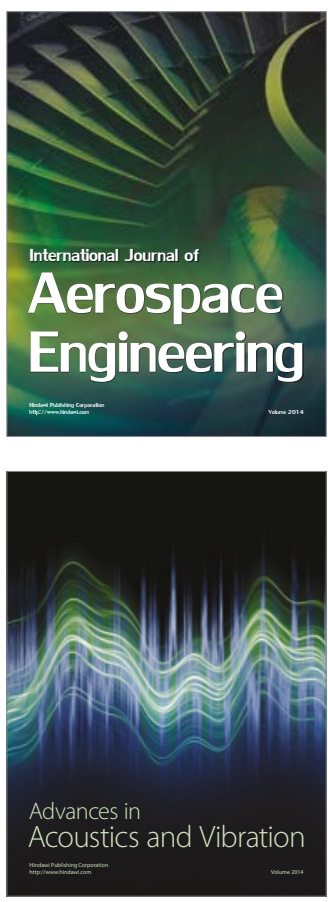

Sensor Networks 